\title{
SPECTRUM CONSERVATION TECHNIQUES \\ FOR FUTURE TELECOMMUNICATIONS
}

\author{
Robert L. Hinkle \\ National Telecommunications and Information Administration \\ 179 Admiral Cochrane Drive \\ Annapolis, MD 21401
}

\section{ABSTRACT}

The radio spectrum is a limited natural resource that must be carefully managed to ensure its availability to accommodate new telecommunication systems in the near future and into the 21 st century. Therefore, NTIA has established, as a major policy initiative within its long-range plan for management of the spectrum, the identification of spectrum-conservation techniques that will ensure more effective and efficient spectrum utilization in the future. Studies carried out by NTIA have identified several frequency bands that are becoming congested and situations where the evolution of new technology to more susceptible equipment has overtaken existing standards. In these situations, to permit continued growth sufficient lead time is required to allow development of economical technology and the establishment of regulatory policy.

This paper provides a quantitative method for evaluating spectrum conservation techniques, an overview of several spectrum conserving technologies and policy/regulatory considerations related to implementing spectrum efficient technologies in the future.

\section{INTRODUCTION}

Several frequency bands have been identified by the National Telecommunications and Information Administration (NTIA) and the Federal Communications Commission (FCC) as being potentially congested within the United States. Similarly, bands have been identified by the ITU (International Telecommunications Union) as being potentially congested internationally.

With increases in demand for spectrum, there has also been an increase in sharing of spectrum among allocated services. An example of this trend towards sharing is the recent FCC ruling on sharing among the Land Mobile Satellite, Aeronautical Mobile Satellite and Maritime Mobile Satellite Services in the band segments around $1.5 / 1.6 \mathrm{GHz}$.

The success of sharing between services depends on a variety of economic and regulatory factors. To ensure effective and efficient use of the spectrum regulatory and policy procedures must be established. However, because of the economic burden that can be associated with requirements for spectrum efficient communication techniques, mandated regulatory controls such as standards should only be implemented where necessary to ensure the continued availability of the spectrum. This encourages effective and economical use of the spectrum, and development of new technologies in a less restrictive regulatory environment, while requiring the application of new spectrum conserving technologies only where necessary. In order to accomplish this policy initiative, NTIA has begun:

1. the development of a method to identify heavily used geographical areas in a band [1-3],

2. the evaluation of available radiocommunication technologies with respect to their ability to conserve the spectrum [4-6],

3. the establishment of spectrum standards to ensure efficient use of the spectrum when necessary.

U.S. Government work not protected by U.S. copyright
These three elements permit the identification of heavily used geographical areas with sufficient lead-time to allow the identification and/or development of appropriate and economical spectrum conservation techniques so that a spectrum crisis does not occur and regulatory and policy options are not limited.

\section{QUANTITATIVE EVALUATION OF SPECTRUM CONSERVATION TECHNIQUES}

To assess the contribution of the various design factors or technological options in achieving spectrum conservation, it is necessary to evaluate, quantitatively, the relative spectrum utilization efficiency. One needs to consider all the system design factors, since optimum spectrum utilization for the system generally cannot be achieved by considering any one factor.

For efficient spectrum utilization, any radiated RF energy should be kept to a minimum level, sufficient to ensure an adequate quality of service. This minimization can be realized considering time, frequency, and space (area) domains. CCIR Report 662 [7] defines spectrum utilization efficiency as the ratio of communications achieved to the spectrum space used. Spectrum space is defined as the product of operating time, bandwidth, and the area denied to other users by potential interference. For this analysis, the equation in CCIR Report 662 was used to define the spectrum-conservation factor (SCF):

$$
\mathrm{SCF}=\text { Communications Achieved } /(T \cdot A \cdot B)
$$

where: $T=$ Percentage of time a system shares the spectrum with other users.

$$
\begin{aligned}
& A=\text { Denial area }\left(\mathrm{km}^{2}\right) \\
& B=\text { Spectrum occupied or denied }(\mathrm{Hz})
\end{aligned}
$$

Communications achieved is a function of several factors: the amount of information transmitted over a single channel (e.g., number of voice channels), the number of users of a single or several channels and the distance over which the information is transferred. Equation (1) was chosen because it takes into account both spectrum and spatial (area) denial in assessing the spectrum-conservation properties of a system. The term "denial area" in Equation (1) is a function of the system antenna-pattern characteristics, transmitter output power and the receiver interference threshold level.

The following is an overview of the relation between the various parameters in Equation 1 and the telecommunication design factors or technological options used to achieve efficient use of the spectrum, and the policy/regulatory aspects of implementing spectrum efficient technologies in the future.

\section{COMMUNICATIONS ACHIEVED}

To achieve higher communication capacity in a given bandwidth or channel it is necessary to improve the system transmission efficiency (voice channels $/ \mathrm{Hz}$ ) or the signals in space must have some unique signal identification characteristic which enables a 413 


\section{Transmission Efficiency}

In recent years, there has been a trend towards higher order digital modulations with higher transmission efficiencies $(\mathrm{b} / \mathrm{s} / \mathrm{Hz})$. TABLE 1 shows the relationship between modulation type, bit rate $(b / s)$, transmission efficiency $(b / s / H z)$, number of voice channels and occupied bandwidth for a $6 \mathrm{GHz}$ common carrier microwave system. The FCC regulates the minimum number of voice channels per carrier channel for the common carrier service [8]; thus limiting the use of spectrum inefficient modulations in some bands. On the other hand, the policy of regulating the communications achieved by a system in a band is one method of improving spectrum efficiency.

The evaluation of spectrum conservation properties for different modulation schemes is very complex in that both spectrum and spatial denial are effected by the choice of modulation type used in a system. In general, system parameters such as occupied bandwidth, required receiver input carrier-to-noise ratio, $(C / N)_{i}$, and maximum permissible interference level are all functions of the modulation type and have a direct bearing on spectrum utilization. However, for each modulation, all these factors are interdependent. For example, decreasing the required occupied bandwidth, B, increases the required $\mathrm{C} / \mathrm{N}$ ratio and vice versa. Thus each factor and its tradeoffs must be considered to determine minimum spectrum use. A study of spectrum conservation techniques for fixed microwave systems which included modulation, antennas and signal processing was conducted by NTIA [6].

\section{Unique Signal Identification Characteristic}

Telecommunication systems designed with unique signal identification characteristics can extract the desired signal through some type of receiver signal processing. Radiodetermination systems generally use pulse signals with unique signal identification characteristics. For pulse signals, receiver signal processing techniques can be used to suppress interference and conserve the spectrum by permitting the operation of several systems on the same frequency or channel. Some interference suppression techniques used in radiodeterminatin systems include: pulse repetition frequency (PRF) discriminators, pulse width discriminators, pulse pair detectors, integrators/enhancers and pulse coding (chirp and phase).

When the $2700-2900 \mathrm{MHz}$ band was identified as a congested band and two major new systems were being planned for the band during the early 1980's, NTIA adopted standards requiring the radar systems to have provisions incorporated to suppress pulsed interference. However, the standard only provides a description of the electromagnetic environment and the required suppression of the interfering signals. This permits the manufacturer to have the flexibility to develop appropriate and economical spectrum conservation techniques.

Another example of using unique signal identification characteristics is the new precision distance measuring equipment (DME/P) system in the 960-1215 MHz band. The DME/P system was a new element of the modern and evolving National Airspace System which placed additional demand on the spectrum allocated to the Aeronautical Radionavigation Service. To accommodate this new system, the International Civil Aviation Organization (ICAO) used pulse pair spacing codes to permit the new system to be overlaid on the existing channels of the distance measuring equipment $(\mathrm{DME} / \mathrm{N})$. Figure 1 shows the signal processing concept of pulse pair discrimination, and TABLE 2 shows the pulse pair spacing channeling plan for $D M E / N$ and DME/P developed by ICAO. To ensure compatibility between the two DME systems, ICAO developed standards and recommended practices (SARPS).

\section{SPECTRUM OCCUPIED OR DENIED}

Both transmitters and receivers can deny spectrum to other potential users. Therefore, transmitter and receiver parameters must both be addressed as part of the regulatory process in promoting efficient use of the spectrum. For transmitters, the key parameters are necessary bandwidth, out-of-band emissions and spurious emissions. For receivers, the efficient use of the spectrum is determined by the RF energy at the receiver input which can be transferred to the receiver intermediate frequency (IF) output or demodulator input.

\section{Necessary Bandwidth}

As mentioned earlier, the modulation type has a direct bearing on system parameters such as the necessary bandwidth, required transmitter output power level, receiver input carrier-to-noise ratio, $(C / N)_{1}$, and maximum permissible interference level (See TABLE 1). The occupied bandwidth $(B)$ for digital modulations is determined by the relationship:

$\mathrm{B}(\mathrm{MHz})=$ Bit Rate $(\mathrm{Mb} / \mathrm{s}) /$ Transmission Efficiency $(\mathrm{b} / \mathrm{s} / \mathrm{Hz})$

TABLE 1 shows an example of the trade-offs of the above parameters as a function of the modulation type. To ensure a just comparison, the analysis is based on theoretical transmission efficiency and receiver input carrier-to-noise ratios for the different modulation types. To evaluate the spectrum-conservation properties of the different modulation types, it was necessary to determine the occupied bandwidth $(B)$ and required transmitter

TABLE 1

\begin{tabular}{|c|c|c|c|c|c|c|c|c|}
\hline \multicolumn{9}{|c|}{ DIGITAL SYSTEM PARAMETERS } \\
\hline $\begin{array}{l}\text { Modulation } \\
\text { Type }\end{array}$ & $\begin{array}{l}\text { Number } \\
\text { of Voice } \\
\text { Channels }\end{array}$ & $\begin{array}{l}\text { Bit Rate } \\
(\mathrm{mb} / \mathrm{s})\end{array}$ & $\begin{array}{l}\text { Transmission } \\
\text { Etficiency } \\
\text { (b/s/Hz) }\end{array}$ & $\begin{array}{l}\text { Occupied } \\
\text { Bandwidth } \\
(\mathrm{MHz})\end{array}$ & $\begin{array}{l}\text { Required } \\
\text { Carrier-to- } \\
\text { Noise } \\
\text { (dB) }\end{array}$ & $\begin{array}{l}\text { Noise } \\
\text { Level } \\
\text { (dBm) }\end{array}$ & $\begin{array}{l}\text { Minimum } \\
\text { Carrier } \\
\text { Level } \\
\text { (dBm) }\end{array}$ & $\begin{array}{c}\text { Transmitter } \\
\text { Power } \\
\text { Level } \\
\text { (dBm) }\end{array}$ \\
\hline 16-QAM & 1344 & 90 & 4 & 22.5 & 21.0 & -96.5 & .75 .5 & 27.5 \\
\hline 64-QAM & 2016 & 175 & 6 & 22.5 & 27.0 & -96.5 & -69.5 & 33.5 \\
\hline 256-QAM & 2688 & 180 & 8 & 22.5 & 33.1 & .96 .5 & -63.4 & 39.6 \\
\hline
\end{tabular}



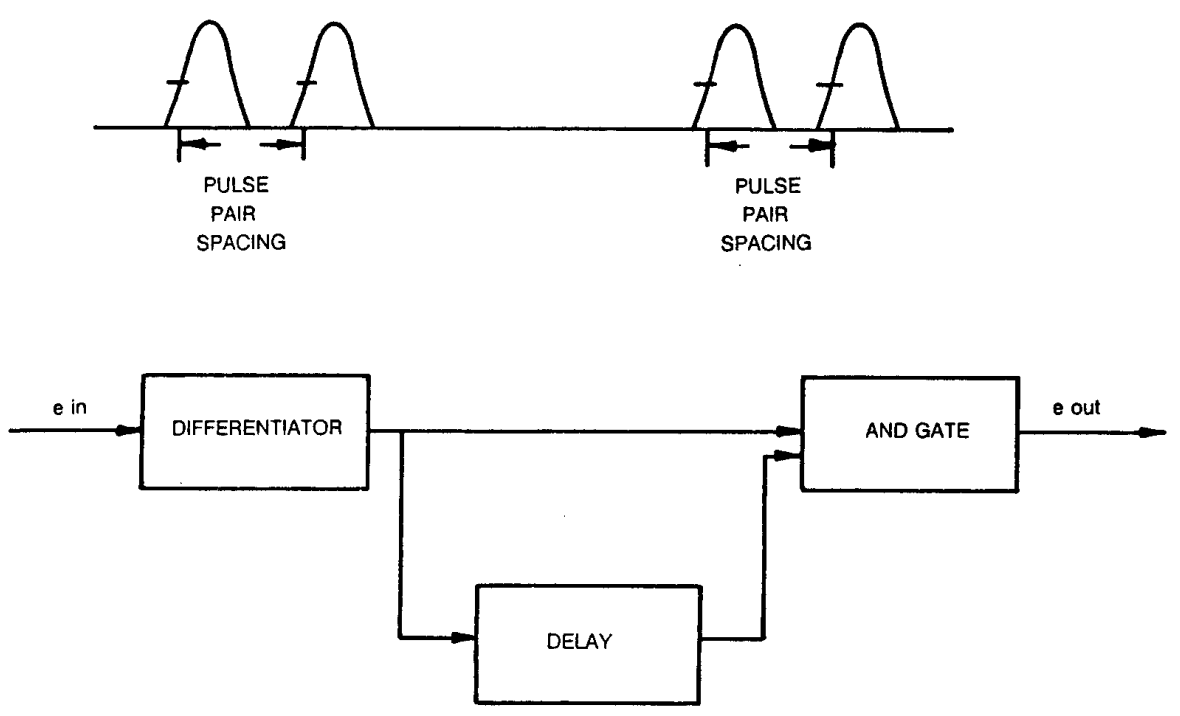

Figure 1. DME Pulse Pair Discrimination Circuit

TABLE 2

PULSE PAIR SPACING FOR DME CHANNEL PLAN

\begin{tabular}{|c|c|c|c|}
\hline \multirow[b]{2}{*}{ Channel } & \multirow[b]{2}{*}{ Operating Mode } & \multicolumn{2}{|c|}{$\begin{array}{l}\text { Pulse Pair } \\
\text { Spacing ( } \mu s)\end{array}$} \\
\hline & & Intent & Reply \\
\hline \multirow[t]{3}{*}{$\mathrm{X}$} & $\mathrm{DME} / \mathrm{N}$ & 12 & 12 \\
\hline & IA Mode & 12 & 12 \\
\hline & FA Mode & 18 & 12 \\
\hline \multirow[t]{3}{*}{$Y$} & $\mathrm{DME} / \mathrm{N}$ & 36 & 30 \\
\hline & IA Mode & 36 & 30 \\
\hline & FA Mode & 42 & 30 \\
\hline \multirow[t]{3}{*}{$\mathbf{W}$} & $\mathrm{DME} / \mathrm{N}$ & - & - \\
\hline & IA Mode & 24 & 24 \\
\hline & FA Mode & 30 & 24 \\
\hline \multirow[t]{3}{*}{$\mathrm{Z}$} & $\mathrm{DME} / \mathrm{N}$ & - & - \\
\hline & IA Mode & 21 & 15 \\
\hline & FA Mode & 27 & 15 \\
\hline
\end{tabular}

Notes:

1. $W$ is a code multiplexed on the $x$ frequency pairing.

2. $Z$ is a code multiplexed on the $Y$ frequency pairing.

3. IA is the initial approach mode (aircraft between 7 and $22 \mathrm{n}$ miles).

4. FA is the final approach mode (aircraft less than $7 \mathrm{n}$ miles). output power level $(P)$ for each. Note that the required transmitter power has a direct bearing on the area denied by a system and will be discussed later.

In recent years, new analog modulation techniques have been developed and studied to assess their spectrum conservation potential. The use of Amplitude Compandored Single Sideband (ACSB) and narrow band frequency modulation (NBFM), $12.5 \mathrm{kHz}$, have been studied as possible solutions to the spectrum congestion in the Government and Non-Government land mobile services in the VHF and UHF bands. One method of achieving efficient modulation techniques is to regulate the channel bandwidth of the allocated services. If regulations are established, providing exclusive spectrum for narrowband technologies, the immediate access to the spectrum will encourage the implementation of spectrum efficient modulations.

\section{Out-of-Band Emissions}

Out-of-band emissions are defined as emissions on a frequency or frequencies immediately outside the necessary bandwidth, resulting from the modulation process but excluding spurious emissions. The out-of-band emissions can be limited through the use of special modulation techniques, such as MSK, and the use of shaping or filtering of the information. However, the spectrum efficiency achieved by these modulation and filtering techniques can only be maintained if the transmitter output device is operated in the linear region. To regulate the out-of-band emissions of a system, emission spectrum masks must be specified.

\section{Spurious Emissions}

Spurious emissions are defined as emissions on a frequency or frequencies immediately outside the necessary bandwidth, the level of which may be reduced without affecting the corresponding transmission of information. Spurious emissions include harmonic emissions, parasitic emissions, intermodulation products and frequency conversion products, but exclude out-of-band emissions. Because spurious emissions can extend for hundreds of $\mathrm{MHz}$, they are extremely important to regulate to ensure efficient use of the spectrum. The spurious emissions are mainly determined by the type of output device used in a transmitter and whether or not the output device is operated in the linear region. The spurious 
emissions of greatest concern are those from high power radiodetermination systems with output powers up to several megawatts. TABLE 3 shows spurious emission characteristics of several types of high power transmitter output devices [9].

TABLE 3

SPURIOUS EMISSION LEVEL RELATIVE TO THE CARRIER MEASURED IN A $1 \mathrm{MHz} B W$

\begin{tabular}{ll} 
& SPURIOUS EMISSION LEVEL \\
& RELATIVE TO THE CARRIER \\
MEASURED IN A $1 \mathrm{MHZ}$ \\
TUBE TYPE & REFERENCE BANDWIDTH (dBC) \\
\hline
\end{tabular}

\section{CROSS FIELD}

Cross Field Amplifiers (CFAs) $\quad-40$ to -70

Magnetrons (unlocked) $\quad-65$ to -80

Magnetrons (locked) $\quad-75$ to -90

Coaxial Magnetrons $\quad-60$ to -75

\section{LINEAR BEAM}

Coupled Cavity TWTS

Klystrons

-105 to -115

-110 to -120

TABLE 3 shows that linear beam tubes have lower spurious emission levels than cross field tubes. However, additional attenuation of spurious emission levels can be achieved through the use of RF bandpass filters. With the use of RF bandpass filters, the spurious emission levels of CFAs, magnetrons and coaxial magnetrons can be reduced below $-100 \mathrm{dBc}$.

The level of permitted spurious emissions should be an absolute level determined by characteristics of systems in the shared band as well as the adjacent bands. The level of permitted spurious emissions should be regulated to ensure efficient use of the spectrum, and included as part of the transmitter emission spectrum mask.

\section{Receiver Parameters}

As mentioned earlier, many receiver parameters are also important in achieving efficient use of the spectrum since receivers can also deny spectrum to other potential users of the spectrum. For receivers, the efficient use of the spectrum is determined by the RF energy at the receiver input which can be transferred to the receiver intermediate frequency (IF) output or demodulator input. Key receiver parameters which determine the transfer of RF energy are:

\footnotetext{
- Receiver Sensitivity

- Receiver RF Selectivity

- Receiver IF Selectivity

- Image and Spurious Response Rejection

- Receiver Desensitization

- Adjacent Channel Rejection
}

It may not be necessary to include all the above receiver performance parameters to ensure efficient use of the spectrum by receivers. The policy aspects of regulating receiver parameters has been controversial since it can be viewed that receivers operate at their own sufferance (i.e., receivers can not cause interference, they can only receive interference). However, in recent years the establishment of receiver standards or the promulgation of receiver guidelines to equipment manufacturers is increasingly being considered by the spectrum management community.
TIME

Equation 1 also considers the percentage of time the system shares the spectrum with other users as a factor in spectrum conservation. However, when a frequency or channel assignment is granted to a user, that frequency or channel is denied to another user within the denied area. For many land mobile operations, the actual use (transmission) on the assigned channel may be a low percentage of time (less than $5 \%$ of the time). Therefore, if a channel can be time-shared, the spectrum can be conserved and used more efficiently.

Trunking is one method of achieving more efficient use of time on land mobile channels. In general, terms, trunking can be described as a communications system that serves more users than there are channels available by sharing common trunk lines (channels) and switching users to available channels using various switching techniques. For example, if one hundred users are assigned to a five channel trunking system, the percentage of time parameter, $T$, in Equation 1 would be .05 .

Until recently, the only trunked land-mobile equipment marketed in the United States operated in the non-government bands, 806$821 \mathrm{MHz}$ and $851-866 \mathrm{MHz}$. NTIA studied the implementation of trunked systems for Federal Goverment use [10], and recently the Department of the Navy implemented two trunked systems operational in the $406.1-420 \mathrm{MHz}$ band. NTIA is currently establishing a shared Government trunked system in the Washington D.C. area. However, to encourage the use of trunked systems it is necessary to establish, through regulations, channels set aside for trunked systems.

Time Division Multiple Access (TDMA) is another method of achieving more efficient use of time using digital techniques. The cellular industry appears destined to implement TDMA, which would result in a three-to-one increase in the number of communication channels available in a given "slice" of spectrum [11]. It should be noted that the increased spectrum conservation of trunked and TDMA techniques could be included in the numerator of equation 1, communication achieved.

Recent tests of a potentially more spectrum efficient technique known as Code Division Multiple Access (CDMA) have apparently been highly successful in the cellular industry. CDMA uses both the time and frequency/spectrum domain to increase spectrum conservation [12].

\section{DENIED AREA}

The denied area is the area in which another system can not operate without degradation in performance below a specified performnace criteria. Some key system design factors that affect denied area are: antenna radiation pattern, transmitter output power and receiver interference threshold.

\section{Antenna Radiation Pattern}

The antenna of a communication system is a key system design factor in addressing spectrum conservation and more specifically denied area. In recent years, significant advances in the antennadesign areas of sidelobe/backlobe reduction and polarization discrimination have provided the capability for enhanced spectrum efficiency in point-to-point microwave radiocommunications. Also, frequency re-use can be achieved by implementing antenna-design spectrum-conservation techniques. For example, the use of spot beams on satellites enhances frequency re-use. A study of spectrum conservation techniques for point-to-point microwave systems [6], determined that the relative improvement in spectrum conservation of the shrouded dish and conical horn reflector antennas over a standard dish antenna could be up to to 150 percent for a shrouded dish and approximately 150 to 1500 percent for a conical horn reflector depending on the type of modulation. The greatest improvement occurred for 256-QAM modulation. 


\section{Transmitter Output Power}

The required transmitter output power is a function of the modulation (See Table 1). However, the use of signal processing techniques can improve the processing gain of a system permitting lower transmitter output power levels for specified receiver output performance criteria. Thus, through the use of signal processing techniques, the transmitter output power can be lowered reducing its spatial (area) denial. Some signal processing techniques commonly used in digital microwave systems are antenna space diversity (10 dB gain), adaptive equalizers (4 $\mathrm{dB}$ gain) and forward error correction, FEC, ( $3 \mathrm{~dB}$ gain for high efficient code rate, 18/19 code rate). Through the use of signal processing techniques, the microwave industry has achieved reliable system performance in higher order digital modulations which require higher transmitter output powers while keeping the transmitter output power at one Watt or below. Also, in Radiodetermination systems, signal processing techniques such as chirped pulses and phase coded pulses can be used to reduce the required peak transmitter power.

\section{SUMMARY}

This paper has provided an overview of several spectrum conserving technologies for radiocommunication services and the policy/regulatory aspects related to implementing spectrum efficient technologies in the future. In summary, there are many trade-offs in communication system design factors and technology options that have an impact on spectrum efficiency. The quantitative assessment of these trade-offs on spectrum conservation are extremely complex. The spectrum conserving potential of a system is a function of several design factors and all of these design factors must be taken into consideration when evaluating the spectrum utilization efficiency of a system. That is, one can not say that a system with a particular modulation is more spectrum conserving than a system with another modulation without considering all of the other design factors such as antennas, signal processing, RF filters, etc.

To ensure effective and efficient use of the spectrum, regulatory and policy procedures must be established. However, because of the economic burden on the market place that can be associated with requirements for spectrum efficient communication techniques, mandated regulatory controls such as standards should only be implemented where necessary to ensure the continued availability of the spectrum. This encourages effective and economical use of the spectrum, development of new technologies in a less restrictive regulatory environment, and the application of new spectrum conserving technologies only where necessary.

\section{FUTURE DIRECTION}

The NTIA will continue to implement regulatory and policy measures to ensure the continued availability of the spectrum to meet future requirements. This effort is envisioned to include:

- The application of policy and techniques to ensure the early identification of potentially congested bands to avoid spectrum crises.

- The early establishment of policy and regulations conducive to the development and deployment of spectrum conservation techniques in bands becoming congested.

- The up-front development of spectrum standards for new services and major systems. This will include more rigorous technical studies to establish appropriate system spectrum standards considering both system performance and economic burden.

- The use of common Government and Non-Government spectrum standards in shared bands.
- The promulgation of receiver guidelines in regulations to ensure that manufacturers of equipment have information to design spectrum efficient receivers.

- The establishment of reserved spectrum through regulations in bands which the usage is decreasing.

\section{REFERENCES}

[1] Mayher, R.J.; Haines, R.H., et al; "The SUM Data Base: A New Measure of Spectrum Use," NTIA Report 88-236, National Telecommunications and Information Administration, Washington, D.C., August 1988.

[2] Mayher, R.J.; Memorandum of July 5, 1989, to holders of NTIA Report 88-236, "The SUM Data Base: A New Measure of Spectrum Use," Subject, Revisions and Additions to the Report.

[3] Haines, R.H. and Letts, S.E.; "The SUM Mobile Model: Application of the Spectrum Use Measure to the land Mobile Service," National Telecommunications and Information Administration, Washington, D.C., September 1989.

[4] Hinkle, R.L., "Background Study on Efficient Use of the 27002900 MHz Band," NTIA Report 83-117, National Telecommunications and Information Administration, Washington, D.C., April 1983.

[5] Shelton, W., and Cohen, D., "Assessment of Narrowband Modulation Technologies for Government Land Mobile Operations", NTIA. Report 84-156, National Telecommunications and Information Administration, Washington, D.C., August 1984.

[6] Hinkle, R.L., "Spectrum-Conservation Techniques for Fixed Microwave Systems, NTIA Report 89-243, National Telecommunications and Information Administration, Washington, D.C., May 1989.

[7] CCIR Report 662-2, Definition of Spectrum Use and Efficiency, Volume 1, International Telecommunication Union, Dubrovnik, 1986.

[8] FCC, Title 47, Part 21, Code of Federal Regulations, October 1987.

[9] Gawthrop, P., Internal NTIA Memorandum on Radiodetermination Output Device Spurious Emission Levels, December 1988.

[10] Speights, W.D., "Application of Trunking to Government LandMobile Communications," NTIA Report 87-121, National Telecommunications and Information Administration, Washington, D.C., February 1987.

[11] Baumgartner, F., "Code Division Multiple Access, Beyond the Time Domain," Communications, February 1990.

[12] Kerver, T., "SMRs: New sophistication in the '90s," Communications, February 1990. 